\title{
sartorius
}

\section{Fast, reproducible and reliable determination of biomass in suspension cell cultures with VoluPAC tubes}

\begin{abstract}
VoluPAC tubes from Sartorius offer a convenient and economical alternative for biomass assessment that is at least five times more accurate and about ten times faster than manual cell counting. This method provides data that can be correlated with parameters like cell count or metabolic activity, and allows for close observation of cell growth kinetics in very short time intervals.
\end{abstract}

When working with cell cultures, the determination of cell number using microscopic techniques is part of the daily routine. The most widespread method for counting cells relies on the hemacytometer. This method is highly subjective, imprecise and also very tedious. Even with careful attention to detail, the overall error rate is typically more than $15 \%$. Moreover, it usually takes 5-15 min to perform a single cell count.

Automated cell counters offer a more precise and faster alternative, whether they are based on digital image recognition, make use of differences in the electrical properties of the cells or require specific dyes and buffers. Nevertheless, these techniques have other drawbacks: they often require additional dilution steps, and problems can occur with cells forming microclusters. They are also regarded as sophisticated and costly techniques that require special user training.

The determination of biomass as an expression of packed cell volume (PCV) is a proven method used by medical laboratories, where PCV is known as hematocrit. Usually, a small sample of blood is centrifuged in a capillary device; blood cells are pelleted within the capillary and the volume of the packed blood cells is determined. Mammalian cells grown in vitro usually do not reach densities conducive to standard hematocrit analysis. The use of this fast and straightforward technique requires the adaptation of these tubes to the requirements of mammalian cell culture.

The VoluPAC tube is similar in size to standard microcentrifuge tubes. It consists of a sample volume reservoir with a capacity of 1,000 $\mu \mathrm{l}$ and a capillary with a maximum volume of $5 \mu \mathrm{l}$ directly attached to the reservoir (Fig. 1). The capillary is graduated in $0.5-\mu l$ steps, allowing for very precise reading of the height of the cell pellet after centrifugation. The PCV is calculated as follows: PCV $(\%)=$ (volume of cell pellet/volume of sample) $\times 100$.

\section{Joachim Luecke}

Sartorius AG, Weenderlandstr. 94-1008, D-37075 Goettingen, Germany. Correspondence should be addressed to J.L. (joachim.luecke@sartorius.com).

PUBLISHED ONLINE 21 SEPTEMBER 2006; DOI:10.1038/NMETH942

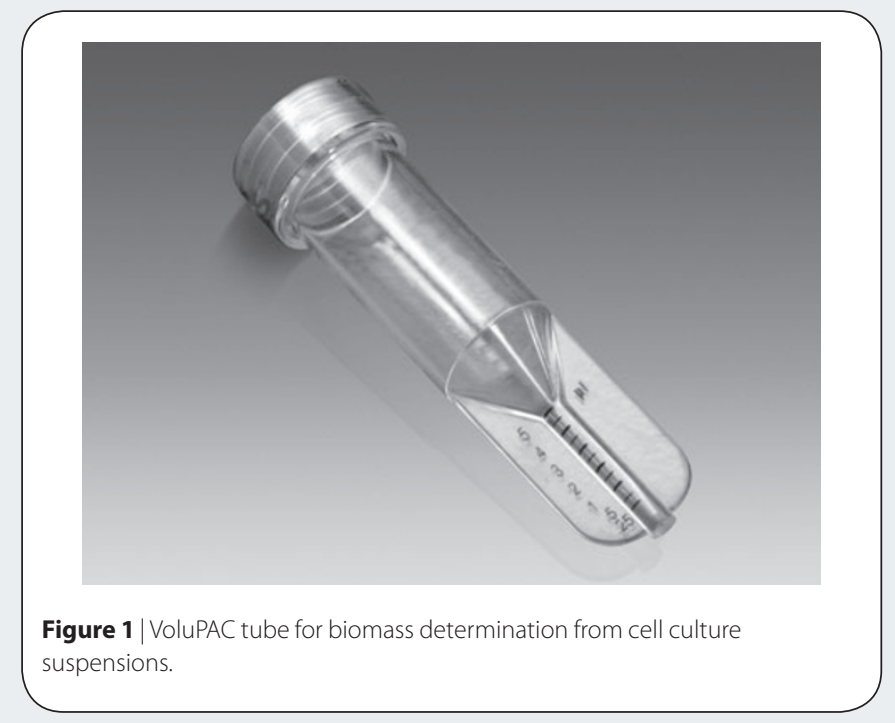

We evaluated the use of VoluPAC tubes for biomass determination in cell culture with suspension-adapted CHO DG44, HEK 293 and NSO cells ${ }^{1}$. These cells were cultivated in cell-appropriate medium in either 50-ml disposable centrifuge tubes or 500-ml round or square bottles, and incubated in a $\mathrm{CO}_{2}$ - and humidity-controlled atmosphere at $37^{\circ} \mathrm{C}$ with orbital agitation. The cells were passaged every $3-4 \mathrm{~d}$ at densities of $2-5 \times 10^{5}$ cells $/ \mathrm{ml}$. For PCV measurements, aliquots of a well-mixed suspension culture $(100-1,000 \mu \mathrm{l}$ in multiples of $100 \mu \mathrm{l})$ were transferred into VoluPAC tubes. The tubes were centrifuged in a microcentrifuge equipped with a swinging bucket rotor for $1 \mathrm{~min}$ at $2,500 \mathrm{~g}$ (5,000 r.p.m.). The height of the cell pellet was visually determined by taking a reading from the capillary graduation; alternatively, an image analysis device was used.

To demonstrate the precision of biomass determination with the VoluPAC tubes, ten individual samples from the same CHO DG44 suspension culture were measured. The PCV determination was then repeated $1 \mathrm{~h}$ later with the same culture. The statistical analysis of the results showed that the standard deviation of the ten independent PCV 
APPLICATION NOTES

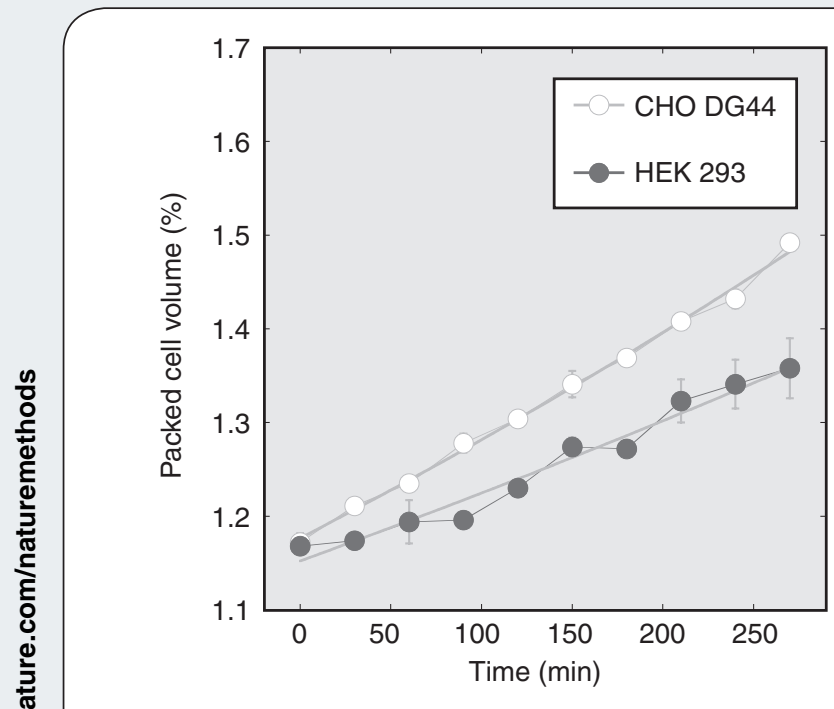

Figure 2 | PCV determination in short time intervals (30 min). Suspension cultures of CHO DG44 and HEK 293 cells were analyzed during exponential growth phase $(n=3)$

measurements was typically less than $1 \%$ of the average value. This indicated a high degree of reliability, superior to data that can be collected by manual counting. The increase of biomass within a period as short as $1 \mathrm{~h}$ was statistically relevant with average PCV values of $0.484 \%$ and $0.492 \%$ for the two time points (data not shown).

To verify the high resolution of the PCV method in respect to biomass increase in short time intervals, exponentially growing $\mathrm{CHO}$ DG44 and HEK 293 cells were cultivated in 500-ml square bottles and samples were taken every 30 min over a period of $4 \mathrm{~h}$. Results showed a measurable increase in biomass from a starting PCV value of $1.17 \%$ for both cell lines up to $1.49 \%$ and $1.36 \%$ for CHO DG44 and HEK 293 cells, respectively. By fitting the data with an exponential curve, the specific growth rates $(\mu)$ were determined to be $8.6 \times 10^{-4}$ and 6.1 $\times 10^{-4} \mathrm{~min}^{-1}$ for CHO DG44 and HEK 293 cells, respectively (Fig. 2). These values corresponded to doubling times of approximately 13 and $19 \mathrm{~h}$, respectively. Neither manual cell counting nor automated counters allow for the measurement of cell growth in such short time intervals.

To determine the correlation between cell number (counted manually) and PCV, samples were taken from CHO DG44 and NSO cultures subcultivated every 3-4 d. Data were assessed in duplicate twice every day for a culture period of $30 \mathrm{~d}$. Figure 3 shows average PCV values plotted against average cell density. The two cell lines have different individual cellular volumes; however, the relationship was approxi-

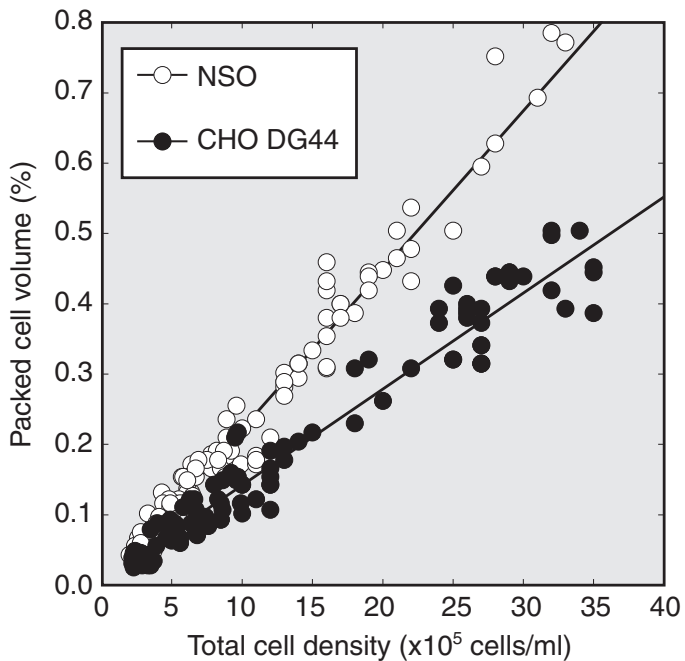

Figure 3 | Correlation between PCV and total cell density, as assessed by manual cell counting. CHO DG44 and NSO myeloma cells were subcultivated every 3-4 d and samples were taken twice per day $(n=2)$.

mately linear up to a density of $4 \times 10^{6}$ cells $/ \mathrm{ml}$. At higher cell densities, the average cell volume was slightly reduced, and the correlation was not linear (data not shown).

These data clearly show that the VoluPAC tubes for biomass determination in suspension cell cultures provide results with very high precision and reliability. Differences in growth kinetics and metabolic activity can be screened with high time resolution. This is not feasible with other presently available techniques such as manual counting with a hemacytometer. Thus, the VoluPAC tubes represent an invaluable tool for monitoring processes in biofermentation of different scales with various cell lines and diverse formulations of media. VoluPAC tubes have also been demonstrated to be applicable to biomass assessment with bacterial and fungal cultures in suspension (data not shown).

\section{ACKNOWLEDGMENTS}

The author thanks M. Jordan, M. Stettler and F. Wurm of the Laboratory of Cellular Biotechnology in the School of Life Sciences of the École Polytechnique Fédérale de Lausanne for their contribution to this work.

1. Stettler, M. et al. New disposable tubes for rapid and precise biomass assessment for suspension cultures of mammalian cells. Biotech. and Bioeng. (in the press) preprint at http://www3.interscience.wiley.com/cgi-bin/ abstract/112729559/ABSTRACT (2006).

This article was submitted to Nature Methods by a commercial organization and has not been peer reviewed. Nature Methods takes no responsibility for the accuracy or otherwise of the information provided. 